\title{
Time-resolved luminescence from Jahn-Teller split states of self-trapped excitons in $\mathrm{PbWO}_{4}$
}

\author{
Minoru Itoh* and Takaaki Sakurai \\ Department of Electrical and Electronic Engineering, Faculty of Engineering, \\ Shinshu University, 4-17-1 Wakasato, Nagano 380-8553, Japan
}

(Received

This paper reports the observation of time-resolved luminescence from a self-trapped exciton (STE) localized at tetrahedral $\left(\mathrm{WO}_{4}\right)^{2-}$ complex anion in $\mathrm{PbWO}_{4}$. The results clearly reveal that the STE luminescence is composed of three emission bands peaking at 407, 425, and $450 \mathrm{~nm}$. Such a composite nature is explained well by assuming that the lowest triplet states of the STE split into three sublevels owing to the symmetry lowering from $T_{\mathrm{d}}$ to $C_{3 \mathrm{v}}$ due to the Jahn-Teller effect. This assumption is in accordance with the theoretical prediction of Bacci et al. The decay curve of each component and its temperature dependence are investigated in the range of 77 to $300 \mathrm{~K}$. These results are discussed on the basis of an adiabatic potential energy diagram of the triplet STE state. Time-resolved luminescence spectra are also measured for Mo-doped $\mathrm{PbWO}_{4}$ crystals. From a comparison with the spectra of undoped $\mathrm{PbWO}_{4}$ crystals, the Mo-related luminescence is clearly separated from the intrinsic luminescence due to STEs.

PACS: 71.35. $-\mathrm{y}, 71.70 .-\mathrm{d}, 78.47 .+\mathrm{p}, 78.55 . \mathrm{Hx}$

*Corresponding author; E-mail address: itohlab@shinshu-u.ac.jp, Phone: +81-26-269-5261, Fax: +81-26-269-5220 
Published in: Phys. Rev. B. 73(23):235106 (8 J une 2006)

\section{INTRODUCTION}

The Jahn-Teller (JT) effect is the intrinsic instability of an electronically degenerate complex against distortions that remove the degeneracy. Since the prediction of JT effect in 1937, ${ }^{1)}$ it has been extensively studied as a very general phenomenon in a wide variety of systems. $^{2,3)}$ A typical example of the JT effect is the impurity center of a heavy metal ion with an outermost shell of (ns) $)^{2}$ configuration in alkali halides, in which the removal of the degeneracy of excited states results in a few minima of the adiabatic potential energy surfaces (APESs) in the configuration coordinate diagram., 5) Another well-known example is the off-center self-trapped exciton (STE) in alkali halide crystals. ${ }^{6)}$ In alkali halides, the STE in its lowest triplet state with $D_{2 \mathrm{~h}}$ symmetry is unstable with respect to an axial displacement of the hole localized on two adjacent halogen ions, resulting in spontaneous relaxation to an off-center configuration having the $C_{2 v}$ local symmetry. This off-center instability of STEs is well explained in terms of the pseudo JT effect. ${ }^{7,8)}$

Lead tungstate $\mathrm{PbWO}_{4}$ (PWO) crystallizes in the scheelite structure, in which $\mathrm{W}$ ions are in tetrahedral $\mathrm{O}$-cages isolated from each other and a $\mathrm{Pb}$ ion is surrounded by eight oxygen ions. The intrinsic luminescence peaking around $425 \mathrm{~nm}$ of PWO has drawn much attention as a promising candidate for scintillation detectors in high-energy particle physics 9 , ${ }^{10)}$ and in positron emission tomography (PET) for medical diagnosis of cancers and other diseases. ${ }^{11,12)}$ This blue luminescence is attributed to the radiative recombination of an STE localized at the tetrahedral $\left(\mathrm{WO}_{4}\right)^{2-}$ molecular ion. ${ }^{13,14)}$ In an isolated $\left(\mathrm{WO}_{4}\right)^{2-}$ site with tetrahedral symmetry $T_{\mathrm{d}}$, four electronic states, two singlets and two triplets, are expected to result from the $\left(t_{1}\right){ }^{5}(e)^{1}$ excited configuration: ${ }^{1} T_{2}>{ }^{1} T_{1}>{ }^{3} T_{2} \cong{ }^{3} T_{1}{ }^{15)}$ In general, it has been accepted ${ }^{16)}$ that the blue luminescence originates from the ${ }^{3} T_{1}$ and (or) ${ }^{3} T_{2} \rightarrow{ }^{1} A_{1}$ transition, which is allowed by the spin-orbit interaction with the high-lying singlet ${ }^{1} T_{1,2}$ states. This model, however, does not take the JT effect into consideration. There are a few papers suggesting some influence of the JT effect on the triplet luminescence in tungstates. ${ }^{17,18)}$ However, no clear experimental evidence for the JT effect has been reported so far, although Polak et al. ${ }^{19)}$ have regarded three-peaked structure in their luminescence spectra of PWO as a favorable manifestation of the JT effect. 
A general theoretical approach to tetrahedral complexes with an excited state consisting of two closely lying triplet states was developed by Bacci et al. ${ }^{20,21)}$ Such a level scheme is practically applicable to the luminescence process of STEs in tungstates. Assuming that the JT interaction is greater than the pseudo JT interaction, they showed that, when the JT effect is considered, the electronic structure of the triplet state becomes complex with a few adiabatic potential energy surfaces. This makes the STE luminescence spectrum composed of several emission bands. It would be very hard to resolve such a composite structure by ordinary luminescence measurements because of the strong spectral overlap of the respective emission bands.

In the present work, we have performed time-resolved luminescence measurements on PWO crystals in the temperature range of $T=77-300 \mathrm{~K}$. The results clearly reveal that the STE luminescence is composed of three emission bands with different decay times, in accordance with the prediction of Bacci et al. Temperature dependence of the decay time of each component is discussed on the basis of a configuration coordinate model of the triplet state. Time-resolved luminescence spectra are also measured for Mo-doped PWO crystals, in order to clarify the nature of the Mo-related luminescence in PWO. A rapid letter of this work has been reported in Ref. 22.

\section{EXPERIMENT}

The single crystals used in the present experiments were prepared at the Materials Research Laboratory of Furukawa Co., Tsukuba. Raw powdered materials $\mathrm{PbO}$ and $\mathrm{WO}_{3}$ of a purity of $99.99 \%$ were purified by the three-time recrystallization technique. The crystals of undoped and Mo-doped (1350 at.ppm) PWO were grown by the Czochralski method using a platinum crucible. The sample was mounted on the copper holder in a vacuum nitrogen cryostat. The temperature of samples was monitored with a copper-constantan thermocouple, and was controlled within $\pm 2 \mathrm{~K}$.

The fourth harmonic (266 nm; $7 \mathrm{ns)} \mathrm{of} \mathrm{a} \mathrm{Q-switched} \mathrm{Nd:YAG} \mathrm{laser} \mathrm{(Continuum} \mathrm{Minilite}$ II) was used as a pulsed light source, which corresponds to the above-gap excitation of PWO. The laser power was approximately $50 \mathrm{~mW} \mathrm{~cm}^{-2}$ on the sample surface. Time-resolved 
luminescence spectra were observed by means of a Jobin-Yvon HR320 monochromator equipped with a gated ICCD camera (Hamamatsu C5909-06), with a spectral resolution of $10 \mathrm{~nm}$. The temporal resolution was changed depending on the decay times of the emission bands. The luminescence spectra reported here were not corrected for the spectral response of the detection system; in fact, it is not so dependent on the wavelength in the spectral region of interest.

\section{RESULTS}

Time-resolved luminescence spectra were measured at several different temperatures in the range of $T=77-300 \mathrm{~K}$. The delay time $\tau_{\mathrm{D}}$ was changed from 0 to $1 \mathrm{~ms}$, with a gate width $\Delta t$ ranging from 50 ns to $80 \mu \mathrm{s}$. The results of undoped PWO obtained at 150, 230, and $300 \mathrm{~K}$ are presented in Figs. 1(a), 1(b), and 1(c), respectively. Each spectrum is vertically displaced for clarity, with a multiplication factor on the left side. The time-integrated luminescence spectra at 150, 230, and $300 \mathrm{~K}$ are also shown in the upper part of the respective figures, for reference.

From Fig. 1(c), it appears that the blue luminescence at $300 \mathrm{~K}$ consists of four emission bands with different decay times. The decomposition into four bands was undertaken as follows: 1) A band peaking at $425 \mathrm{~nm}$ is observed just after the pulse excitation $\left(\tau_{\mathrm{D}}=0\right)$, which is labeled II (blue). 2) A band is observed around $480 \mathrm{~nm}$ when $\tau_{\mathrm{D}}$ is as long as $1 \mathrm{~ms}$, and is labeled IV (green). 3) An isolated band appears at $407 \mathrm{~nm}$ when $\tau_{\mathrm{D}}=1$ and $50 \mu \mathrm{s}$, which is labeled I (violet). 4) A band centered at around $450 \mathrm{~nm}$, labeled III (light blue), is desired to achieve a good agreement with the experimental data, especially in the range of $\tau_{\mathrm{D}}$ $=400 \mathrm{~ns}-1 \mu \mathrm{s}$. The sum of these four bands reproduces well the experimental data, as indicated by the red curves.

The decomposition of the time-resolved spectra at $230 \mathrm{~K}$ (Fig. 1(b)) brings us similar results to those at $300 \mathrm{~K}$; i.e., four emission bands I, II, III, and IV are observed. A main difference between 230 and $300 \mathrm{~K}$ is that the bands I and III become observable later in (b) compared with (c). For example, the band I begins to appear at $\tau_{\mathrm{D}}=5 \mu \mathrm{s}$ in (b), while it is observable at $\tau_{\mathrm{D}}=1 \mu \mathrm{s}$ in (c). 
Figure 1(a) reveals that the time-resolved spectra at $150 \mathrm{~K}$ are decomposed into three bands, I, II, and IV, in the range of $\tau_{\mathrm{D}}=0-1 \mathrm{~ms}$ available in our detection system. The main band II has a long decay time at low temperatures ( $T \leq 150 \mathrm{~K})$, as shown below. Consequently, the band III is completely masked by the band II at $150 \mathrm{~K}$. It is to be noted that the time-integrated luminescence spectrum at $300 \mathrm{~K}$ has a peak at around $425 \mathrm{~nm}$, which is blue-shifted by about $10 \mathrm{~nm}$ relative to that observed at $150 \mathrm{~K}$ or less.

The emission intensity of the decomposed bands I, II, and III in undoped PWO was measured by changing $\tau_{\mathrm{D}}$ while keeping $\Delta t$ at a constant value. From this measurement, we got the decay curves of the respective bands separately. For the band II, it was found that the initial intensity at $t=0$ is almost independent of the temperature. Figure 2 shows the decay curves of the decomposed band II at five different temperatures. One may see that all the decay curves are non-exponential, and are fitted by a sum of three components; $I(t)=$ $I_{\mathrm{f}}(0) \exp \left(-t / \tau_{\mathrm{f}}\right)+I_{\mathrm{m}}(0) \exp \left(-t / \tau_{\mathrm{m}}\right)+I_{\mathrm{s}}(0) \exp \left(-t / \tau_{\mathrm{s}}\right)$. For instance, the decay curve at $77 \mathrm{~K}$ consists of a fast component $\tau_{\mathrm{f}}=0.4 \pm 0.1 \mu \mathrm{s}$, a medium component $\tau_{\mathrm{m}}=3.0 \pm 0.5 \mu \mathrm{s}$, and a slow component $\tau_{\mathrm{s}}=70 \pm 20 \mu \mathrm{s}$.

The decay kinetics of the main band peaking at $425 \mathrm{~nm}$ has been investigated under different power density of laser light in a wide temperature range 8-300 K. ${ }^{23}$ When the sample is excited by $266-\mathrm{nm}$ photons with a low density $\left(<0.6 \mathrm{~mW} \mathrm{~cm}^{-2}\right)$, the decay curve is well described by a single exponent at low temperatures, but the decay kinetics becomes non-exponential under high-density excitation $\left(60 \mathrm{~mW} \mathrm{~cm}^{-2}\right)$. The present experiments were performed under the condition of high-density excitation. The obtained values of $\tau_{\mathrm{f}}$ and $\tau_{\mathrm{m}}$ are fairly consistent with the previous results observed under high-density excitation by using a photomultiplier and an oscilloscope. ${ }^{23)}$ The slow component is by about 10 times longer than the previous value $(5.6 \mu \mathrm{s})$. The present value seems to be more reliable, because the measurements were done in a wider range of time after the pulse excitation, owing to high sensitivity of the gated ICCD camera used. Temperature dependences of $\tau_{\mathrm{f}}$, $\tau_{\mathrm{m}}$, and $\tau_{\mathrm{s}}$ are presented in Fig. 3. The decay times of all the components rapidly shorten above $150 \mathrm{~K}$. It should be especially noticed that the value of $\tau_{\mathrm{s}}$ decreases from $70 \mu \mathrm{s}$ at 77 $\mathrm{K}$ to $50 \mu$ s at $150 \mathrm{~K}$ to $2 \mu$ s at $230 \mathrm{~K}$ and eventually to $0.2 \mu$ s at $300 \mathrm{~K}$. 
Published in: Phys. Rev. B. 73(23):235106 (8 J une 2006)

Figure 4 shows the decay curves of the band I at four different temperatures. At $150 \mathrm{~K}$, the decay data are missing in the initial stage of $\tau_{\mathrm{D}}(0-20 \mu \mathrm{s})$, because of the masking by the band II. The band I exhibits a single exponential decay. It is seen that the decay time hardly depends on $T$, and is nearly $50 \mu$ s. The initial intensity of the band I extrapolated to $t=0$ did not change with temperature in the range $T=150-300 \mathrm{~K}$.

Figure 5 shows the decay curves of the band III at $T=170,230$, and $300 \mathrm{~K}$. At $170 \mathrm{~K}$, the decay data are again missing in the initial stage of $\tau_{\mathrm{D}}$ for the same reason as the band $\mathrm{I}$. The band III decays single-exponentially, with a decay time $\tau=8 \pm 2 \mu \mathrm{s}$ at $170 \mathrm{~K}, 2 \pm 0.5 \mu \mathrm{s}$ at $230 \mathrm{~K}$, and $0.3 \pm 0.1 \mu$ s at $300 \mathrm{~K}$ (see the inset in Fig. 5). The initial intensity of the band III extrapolated to $t=0$ was almost constant when the temperature was increased from 170 to $300 \mathrm{~K}$.

We also examined time-resolved luminescence spectra of Mo-doped PWO. The result at $300 \mathrm{~K}$ is shown in Fig. 6, as a typical example. The fundamental characteristics of the time-resolved spectra of Mo-doped PWO are very similar to those of undoped PWO; that is to say, the three bands I, II, and III are well decomposed. A remarkable difference is found for the band IV, which corresponds to the longest-lived component. The band IV has a peak at around $480 \mathrm{~nm}$ in undoped PWO, while it has a peak around $540 \mathrm{~nm}$ in Mo-doped PWO. In this connection, the peak position of the time-integrated spectrum of Mo-doped PWO slightly red-shifts, accompanied by an enhanced long-wavelength tail, as compared to the time-integrated spectrum of undoped PWO (Fig. 1(c)).

\section{DISCUSSION}

From Fig. 3 one can recognize that the central band II has a slow decay component of $\tau_{\mathrm{s}} \approx$ $70 \mu \mathrm{s}$ at low temperatures. The decay time $\tau_{\mathrm{s}}$ decreases sharply above $150 \mathrm{~K}$. At $150 \mathrm{~K}$, the value of $\tau_{\mathrm{s}}$ is comparable with the decay time of the band I, but still longer than the decay time of the band III. This is the reason why the band III is not observed in Fig. 1(a). At $T$ $\geq 230 \mathrm{~K}$, the value of $\tau_{\mathrm{s}}$ becomes comparable with or shorter than the decay times of both bands I and III. As a result, the three emission bands I, II, and III are well resolved in Figs. 1(b) and 1(c). It is therefore concluded that the time-resolved measurements at $T>150 \mathrm{~K}$ 
are crucial to the successful observation of the composite nature of the triplet luminescence in PWO. In fact, no appreciable changes in spectral shape and peak position were observed at $77 \mathrm{~K}$ in the range of $\tau_{\mathrm{D}}=0-1 \mathrm{~ms}$.

Let us consider the origin of the three emission bands I, II, and III. The origin of the long-lived band IV will be discussed later. As mentioned in Introduction, the intrinsic luminescence of PWO is ascribed to the radiative decay of an STE localized at tetrahedral $\left(\mathrm{WO}_{4}\right)^{2-}$ complex anion. In free $\left(\mathrm{WO}_{4}\right)^{2-}$ molecule of $T_{\mathrm{d}}$ symmetry, the ground state ${ }^{1} A_{1}$ is derived from the one-electron configuration $\left(t_{1}\right)^{6}(e)^{0} .{ }^{15,20)}$ The lowest excited state of the electronic configuration $\left(t_{1}\right)^{5}(e)^{1}$ consists of the ${ }^{3} T_{1}$ and ${ }^{3} T_{2}$ states separated by an energy gap $\Delta$. Both these levels are degenerate, so that they are JT unstable with respect to the lattice deformations. Because of the symmetry lowering from $T_{\mathrm{d}}$ to $C_{3 \mathrm{v}}$ due to the JT effect, the ${ }^{3} T_{1}$ state splits into the ${ }^{3} A_{2}$ and ${ }^{3} E$ sublevels, and the ${ }^{3} T_{2}$ state into the ${ }^{3} A_{1}$ and ${ }^{3} E$ sublevels. The situation is illustrated schematically in Fig. 7(a). In this figure, we cannot say anything about the energy ordering of each level from the group theoretical consideration.

The energy gap $\Delta$ is expected to be very small, because, to a first approximation, the only difference between the ${ }^{3} T_{1}$ and ${ }^{3} T_{2}$ states is due to Coulomb integrals involving the $2 p$ oxygen orbitals. $^{20)}$ In such a case, the energy level scheme changes from (a) to (b) in Fig. 7. Consequently, it is supposed that the JT distortion of $\left(\mathrm{WO}_{4}\right)^{2-}$ ion results in three APESs of the triplet STE state. The STE luminescence of PWO would arise from the three minima of these APESs, leading to a luminescence band consisting of a triplet structure. This is consistent with the present observation of three emission bands I, II, and III. In Mo-doped samples, the same bands I, II, and III are also observed as shown in Fig. 6, which is additional evidence for the intrinsic nature of these three emission bands.

We make in brief a quantitative comparison between the experiment and the theory. For the separation in peak energy between the bands I and II, we get $\Delta_{\mathrm{I}-\mathrm{II}}=0.13 \mathrm{eV}$ from Fig. 1 , and $\Delta_{\text {II-III }}=0.16 \mathrm{eV}$ for the separation between the bands II and III. Bacci et al. ${ }^{20)}$ have estimated to be $\Delta_{\text {I-II }} \approx 0.15 \mathrm{eV}$ and $\Delta_{\text {II-III }} \approx 0.16 \mathrm{eV}$ from their calculated luminescence spectra. The experimental values are in good agreement with the theoretical estimation. According to the model by Bacci et al., the intensities of the emission bands are affected by 
the value of $\Delta$ and the strength of pseudo JT coupling. When $\Delta \approx 0$ and the pseudo JT coupling is not present, the intensity of the central band II is anticipated to be highly dominant. Their prediction is also in accordance with our result.

All the three emission bands I, II, and III decay with long lifetimes of the order of several tens of $\mu$ s at low temperatures. This is a characteristic feature expected for the triplet luminescence. The main band II exhibits the non-exponential decay kinetics. There are two possible explanations for such decay kinetics. The photo-excitation at $266 \mathrm{~nm}$ creates electrons and holes, and they are separated in space and stored in traps, as pointed out by Mürk et al. ${ }^{24)}$ This suggests that the decay kinetics is “spoilt” by slow delayed recombination phenomena, resulting in the non-exponential decay due to the coexistence of prompt and delayed recombination processes. Another explanation is that the high-density excitation makes nonradiative Auger process of STEs possible, in which one STE recombines nonradiatively, and transfers its energy to another STE, which is consequently decomposed into a free electron and hole. ${ }^{23)}$ Such a nonradiative process causes the shortening of the decay time, i.e., non-exponential decay of the luminescence. Even in either case, their effects would contribute mainly to the emitting II state, because the population responsible for the band II is significantly larger than those of the bands I and III.

The decay times of the bands II and III decrease at high temperatures ( $T>150 \mathrm{~K}$ ), while that of the band I remains almost constant when $T$ is increased. The decrease of the decay time is attributed to the thermal quenching or disintegration of STE. ${ }^{23,24)}$ The present results may be explained on the basis of the APESs depicted schematically in Fig. 8. From Figs. 3 and 5 it is seen that the decay time of the band II decreases in parallel with that of the band III in the range of $T \geq 170 \mathrm{~K}$. This fact suggests that a quenching barrier $E_{\mathrm{II}}$ for the central APES (II) is comparable to the barrier $E_{\text {III }}$ for the lowest APES (III). On the other hand, the barrier height $E_{\mathrm{I}}$ for the highest APES (I) would be greater than the other two. If this speculation is correct, the quenching from the central and lowest APESs is more dominant and thus the decrease of the decay times of the bands II and III becomes efficient at high temperatures.

The peak position of the time-integrated luminescence spectrum of PWO apparently shifts 
toward the short-wavelength side as $T$ is increased. Such blue-shift is unusual in many luminescent systems, where the peak position rather red-shifts with increasing $T$ The present peak shift is also understood in terms of the configuration coordinate diagram depicted in Fig. 8. The central band II is dominant at low temperatures, but it decreases in intensity, as well as the band III, at $T \geq 170 \mathrm{~K}$ due to the thermal quenching. On the other hand, the intensity of the high-energy band I remains constant even at high temperatures, thus resulting in the blue-shift of the luminescence peak. Similar blue-shift of the triplet luminescence band with increasing $T$ has also been observed in other tungstates such as $\mathrm{CdWO}_{4}{ }^{25)}$ and $\mathrm{CaWO}_{4}{ }^{26)}$

It has been found ${ }^{27)}$ that the STE luminescence (probably, the main band II) of PWO shows an order of magnitude increase of the decay time at very low temperatures $(T<5 \mathrm{~K})$. This fact suggests the presence of a pair of closely spaced energy levels involved in the excited-state dynamics of $\left(\mathrm{WO}_{4}\right)^{2-}$ center. The energy distance $\delta$ separating both levels is estimated to be $0.45 \mathrm{meV}$. The origin of these two states has not been discussed in Ref. 27 . Based on the present work, we suppose that they may correspond to the nearly degenerate ${ }^{3} A_{2}$ and ${ }^{3} E$ sublevels shown in Fig. 7(b). The ${ }^{3} A_{2}$ and ${ }^{3} E$ states are accidentally degenerate for $\Delta=0,{ }^{20)}$ but such degeneracy might be lifted by some perturbation such as the crystal field. If so, the magnitude of the energy separation $\delta$ is expected to be sensitively dependent on the crystal structure of tungstates. This expectation is supported by the recent experiment by Babin et al., ${ }^{28)}$ who investigated the luminescence decay kinetics of scheelite-structured PWO and wolframite-structured $\mathrm{CdWO}_{4}$ in the range of $T=0.4-400 \mathrm{~K}$ and revealed that the value of $\delta$ in wolframite is 3-4 times as large as that in scheelite.

As reviewed by Nikl, ${ }^{14)}$ some papers have been reported on the origin of the long-lived band IV in PWO. There is a consensus that this luminescence is extrinsic feature of PWO and arises from the radiative recombination of relaxed excitons perturbed by nearby lattice imperfections. The nature of the lattice imperfection has still been unclear, however. There are four proposals for the imperfection: (i) oxygen-deficient center $\mathrm{WO}_{3}$, possibly with F center nearby, ${ }^{10,13)}$ (ii) local raspite-type distortion, ${ }^{29,}{ }^{30)}$ (iii) complex center $\mathrm{Pb}^{3+}-\left(\mathrm{WO}_{4}\right)^{3-}$, 31) and (iv) Mo impurity center $\left(\mathrm{MoO}_{4}\right)^{2-}{ }^{2-34)}$ In the present experiment, we 
observed the long-lived band IV in Mo-doped PWO, as well. This band is undoubtedly ascribed to the excitons localized at $\left(\mathrm{MoO}_{4}\right)^{2-}$ centers in PWO. ${ }^{35,36)}$ It is interesting to note that the peak position of the band IV in undoped PWO clearly differs from that in Mo-doped PWO. Such a difference distinctly rules out the proposal (iv). The present result says nothing as to which of the other three is correct, though there is a recent paper claiming that the proposal (ii) is improbable. ${ }^{28)}$ The nature of the imperfection responsible for the green band IV in PWO is an open problem to be solved in the near future.

\section{SUMMARY}

From the present experiment, we got clear evidence that the JT effect plays a significant role for the triplet states of an STE localized at $\left(\mathrm{WO}_{4}\right)^{2-}$ center in PWO. Since the main band II has a long lifetime at low temperatures, time-resolved measurements at $T>150 \mathrm{~K}$ were crucial to the successful observation of the composite bands I, II, and III. The experimental results were in satisfactory agreement with the theoretical approach to the tetrahedral complexes with an excited state consisting of two closely lying triplet states. The configuration coordinate model was introduced to explain the temperature dependence of the decay times of the three emission bands I, II, and III, as well as the commonly observed blue-shift of tungstate luminescence. The origin of the long-lived band IV in undoped PWO was also considered in comparison with the results in Mo-doped PWO.

\section{ACKNOWLEDGMENTS}

The authors are grateful to Dr. Y. Usuki for kindly providing them the single crystals used in the present experiments. This work was supported by a Grant-in-Aid from the Ministry of Education, Culture, Sports, Science, and Technology of Japan. 
Published in: Phys. Rev. B. 73(23):235106 (8 J une 2006)

\section{References}

1) H. A. Jahn and E. Teller, Proc. Roy. Soc. A, 161, 220 (1937).

2) M. D. Sturge, in Solid State Physics, Vol. 20, edited by F. Seitz, D. Turnbull, and H. Ehrenreich (Academic Press, New York, 1967) p. 91.

3) Y. Toyozawa, Optical Processes in Solids (Cambridge Univ. Press, Cambridge, 2003) p. 53.

4) Y. Toyozawa and M. Inoue, J. Phys. Soc. Jpn., 21, 1663 (1966).

5) K. Cho, J. Phys. Soc. Jpn., 25, 1372 (1968).

6) K. S. Song and R. T. Williams, Self-Trapped Excitons (Springer-Verlag, Berlin, 1993).

7) Y. Toyozawa, J. Phys. Soc. Jpn., 44, 482 (1978).

8) C. H. Leung and K. S. Song, Phys. Rev. B, 18, 922 (1978).

9) M. Kobayashi, M. Ishii, Y. Usuki, and H. Yahagi, Nucl. Instrum. Methods Phys. Res. A, 333, 429 (1993).

10) P. Lecoq, I. Dafinei, E. Auffray, M. Schneegans, M. V. Korzhik, O. V. Missevitch, V. B. Pavlenko, A. A. Fedorov, A. N. Annenkov, V. L. Kostylev, and V. D. Ligun, Nucl. Instrum. Methods Phys. Res. A, 365, 291 (1995).

11) W. W. Moses, in Proceedings of the Fifth International Conference on Inorganic Scintillators and Their Applications, edited by V. Mikhailin, Moscow State University, 2000, p. 11.

12) M. Kobayashi, Y. Usuki, M. Ishii, M. Itoh, and M. Nikl, Nucl. Instrum. Methods Phys. Res. A, 540, 381 (2005).

13) J. A. Groenink and G. Blasse, J. Solid State Chem., 32, 9 (1980).

14) M. Nikl, Phys. Status Solidi A, 178, 595 (2000).

15) Y. Zhang, N. A. W. Holzwarth, and R. T. Williams, Phys. Rev. B, 57, 12738 (1998).

16) G. Blasse, Struct. Bonding, 42, 1 (1980).

17) Cz. Koepke, A. J. Wojtowicz, and A. Lempicki, J. Lumin., 54, 345 (1993).

18) J. van Tol and J. H. van der Waals, Molec. Phys., 88, 803 (1996).

19) K. Polak, M. Nikl, K. Nitsch, M. Kobayashi, M. Ishii, Y. Usuki, and O. Jarolimek, J. 
Published in: Phys. Rev. B. 73(23):235106 (8 J une 2006)

Lumin., 72-74, 781 (1997).

20) M. Bacci, S. Porcinai, E. Mihóková, M. Nikl, and K. Polák, Phys. Rev. B, 64, 104302 (2001).

21) M. Bacci, E. Mihóková, and L. S. Schulman, Phys. Rev. B, 66, 132301 (2002).

22) M. Itoh and T. Sakurai, Phys. Status Solidi B, 242, R52 (2005).

23) M. Itoh, M. Horimoto, and M. Fujita, J. Phys.: Condens. Matter, 15, 193 (2003).

24) V. Mürk, M. Nikl, E. Mihoková, and K. Nitsch, J. Phys.: Condens. Matter, 9, 249 (1997).

25) M. J. J. Lammers, G. Blasse, and D. S. Robertson, Phys. Status Solidi A, 63, 569 (1981).

26) V. B. Mikhailik, H. Kraus, D. Wahl, M. Itoh, M. Koike, and I. K. Bailiff, Phys. Rev. B, 69, 205110 (2004).

27) M. Nikl, P. Bohacek, E. Mihokova, M. Kobayashi, M. Ishii, Y. Usuki, V. Babin, A.

Stolovich, S. Zazubovich, and M. Bacci, J. Lumin., 87-89, 1136 (2000).

28) V. Babin, P. Bohacek, E. Bender, A. Krasnikov, E. Mihokova, M. Nikl, N. Senguttuvan,

A. Stolovits, Y. Usuki, and S. Zazubovich, Radiat. Meas., 38, 533 (2004).

29) D. L. Alov and S. I. Rybchenko, Mater. Sci. Forum, 239-241, 279 (1997).

30) M. Itoh and M. Fujita, Phys. Rev. B, 62, 12825 (2000).

31) W. van Loo, Phys. Status Solidi A, 28, 227 (1975).

32) M. Kobayashi, M. Ishii, K. Harada, Y. Usuki, H. Okuno, H. Shimizu, and T. Yazawa, Nucl. Instrum. Methods Phys. Res. A, 373, 333 (1996).

33) A. N. Annenkov, E. Auffray, A. E. Borisevich, G. Yu. Drobyshev, A. A. Fedorov, O. V.

Kondratiev, M. V. Korzhik, P. Lecoq, V. D. Ligun, O. V. Missevitch, V. B. Pavlenko, J. -P.

Peigneux, Yu. D. Prokoshkin, A. V. Singovski, and R. F. Zouevski, Nucl. Instrum.

Methods Phys. Res. A, 403, 302 (1998).

34) H. Alves, A. Hofstaetter, F. Leiter, B. K. Meyer, N. G. Romanov, R. Novotny, and M. V. Korzhik, Radiat. Meas., 33, 641 (2001).

35) M. Böhm, A. E. Borisevich, G. Yu. Drobychev, A. Hofstaetter, O. V. Kondratiev, M. V. Korzhik, M. Luh, B. K. Meyer, J. P. Peigneux, and A. Scharmann, Phys. Status Solidi A, 167, 243 (1998).

36) M. Nikl, P. Bohacek, E. Mihokova, N. Solovieva, A. Vedda, M. Martini, G. P. Pazzi, P. 
Published in: Phys. Rev. B. 73(23):235106 (8 J une 2006)

Fabeni, and M. Kobayashi, J. Appl. Phys., 91, 2791 (2002). 
Published in: Phys. Rev. B. 73(23):235106 (8 J une 2006)

\section{Figure captions}

Fig. 1. (Color online) Time-resolved luminescence spectra of PWO at (a) $150 \mathrm{~K}$, (b) $230 \mathrm{~K}$, and (c) $300 \mathrm{~K}$. Each spectrum (delay time: $\tau_{\mathrm{D}}$, gate width: $\Delta t$ ) is vertically displaced for the sake of clarity, with a multiplication factor indicated on the left side. The bands I, II, III, and IV are depicted by violet (dotted), blue (solid), light blue (broken), and green (solid) lines, respectively. The sum of these bands is indicated by red solid line. Time-integrated luminescence spectra are also shown in the upper part of the respective figures.

Fig. 2. Decay kinetics of the band II at various temperatures. Each curve is vertically displaced for clarity. The lines are the best fit of the three-component decay curve to the data points.

Fig. 3. Temperature dependence of the decay times of the band II. The fast, medium, and slow decay times are indicated by open squares, closed circles, and open circles, respectively.

Fig. 4. Decay kinetics of the band I at various temperatures. Each curve is vertically displaced for clarity.

Fig. 5. Decay kinetics of the band III at various temperatures. Each curve is vertically displaced for clarity. The inset shows the temperature dependence of the decay time, from which the thermal activation energy is estimated to be $0.1 \mathrm{eV}$.

Fig. 6. (Color online) Time-resolved luminescence spectra of Mo-doped PWO at $300 \mathrm{~K}$. Each spectrum (delay time: $\tau_{\mathrm{D}}$, gate width: $\Delta t$ ) is vertically displaced for the sake of clarity, with a multiplication factor. The bands I, II, III, and IV are depicted by violet (dotted), blue (solid), light blue (broken), and green (solid) lines, respectively. The sum of these 
Published in: Phys. Rev. B. 73(23):235106 (8 J une 2006)

bands is indicated by red solid line. Time-integrated luminescence spectrum is also shown in the upper part of the figure.

Fig. 7. Energy level diagrams of the triplet STEs in PWO, showing the JT splitting of the ${ }^{3} T_{1}$ and ${ }^{3} T_{2}$ states separated by an energy gap $\Delta$ : (a) $\Delta \neq 0$ and (b) $\Delta \approx 0$.

Fig. 8. Schematic adiabatic potential energy surfaces of the triplet STEs in PWO. The diagram displays the situation that the energy barrier for thermal quenching is in the order of $E_{\mathrm{I}}>E_{\mathrm{II}} \approx E_{\mathrm{III}}$. 

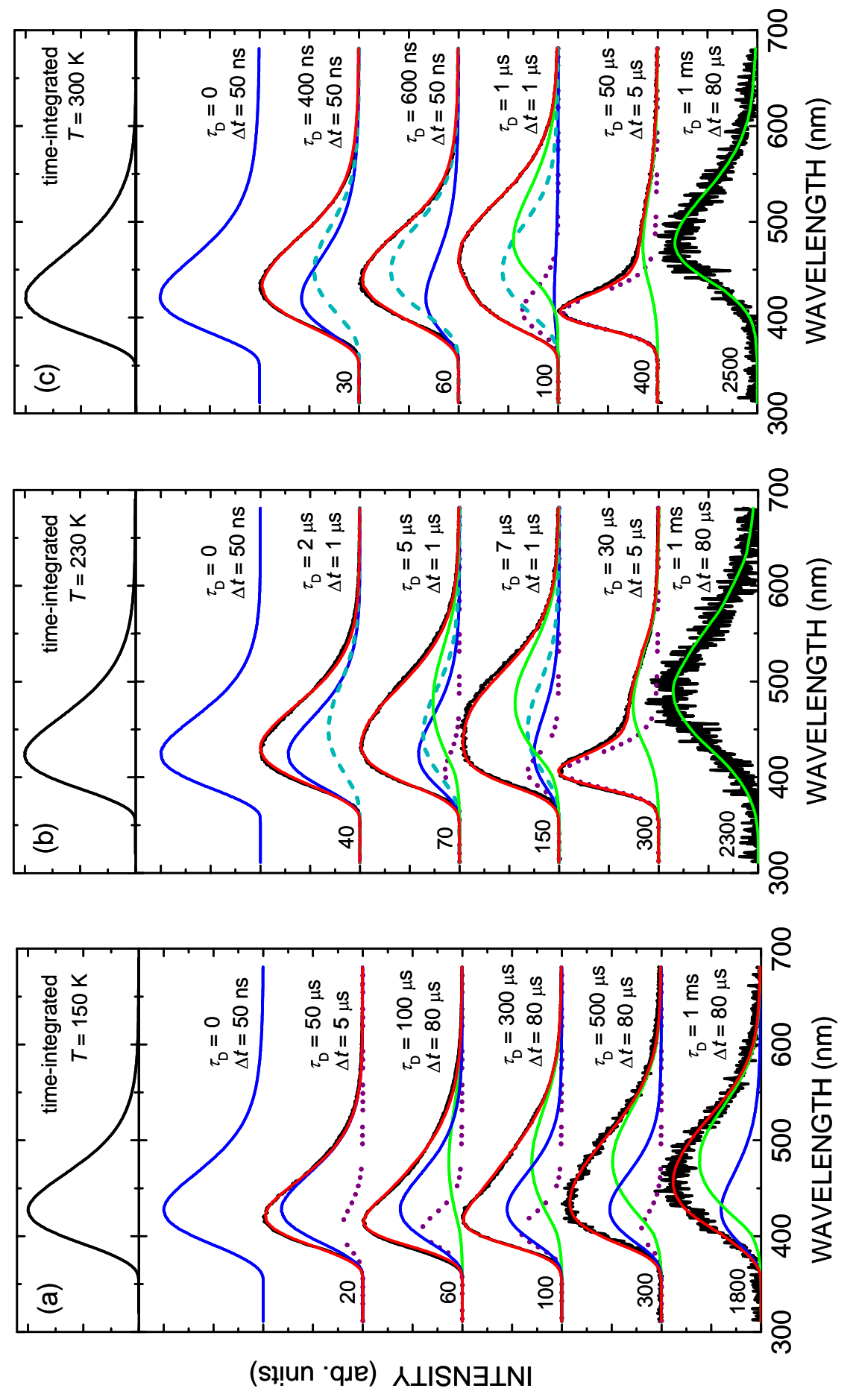

Fig. 1 


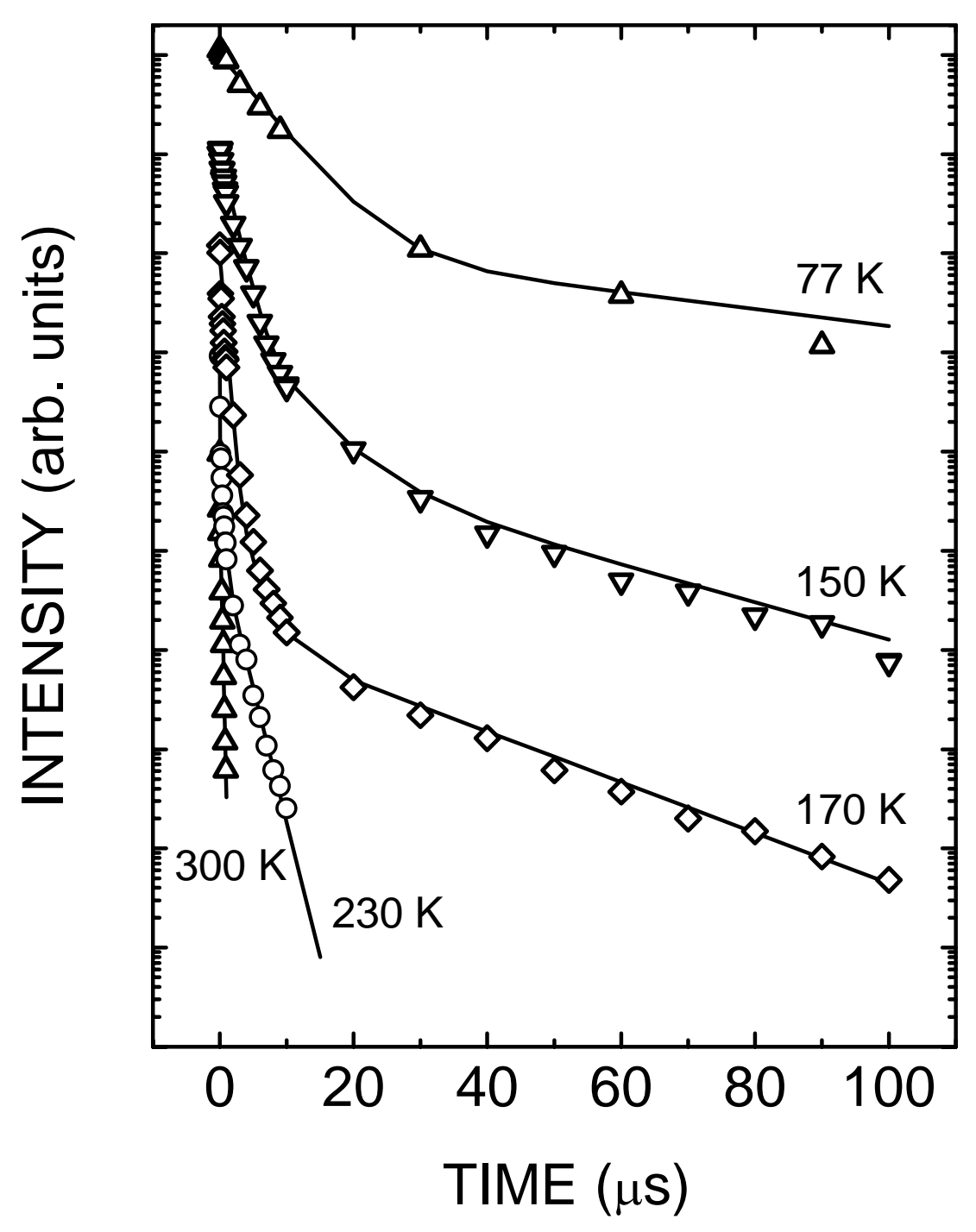

Fig. 2 


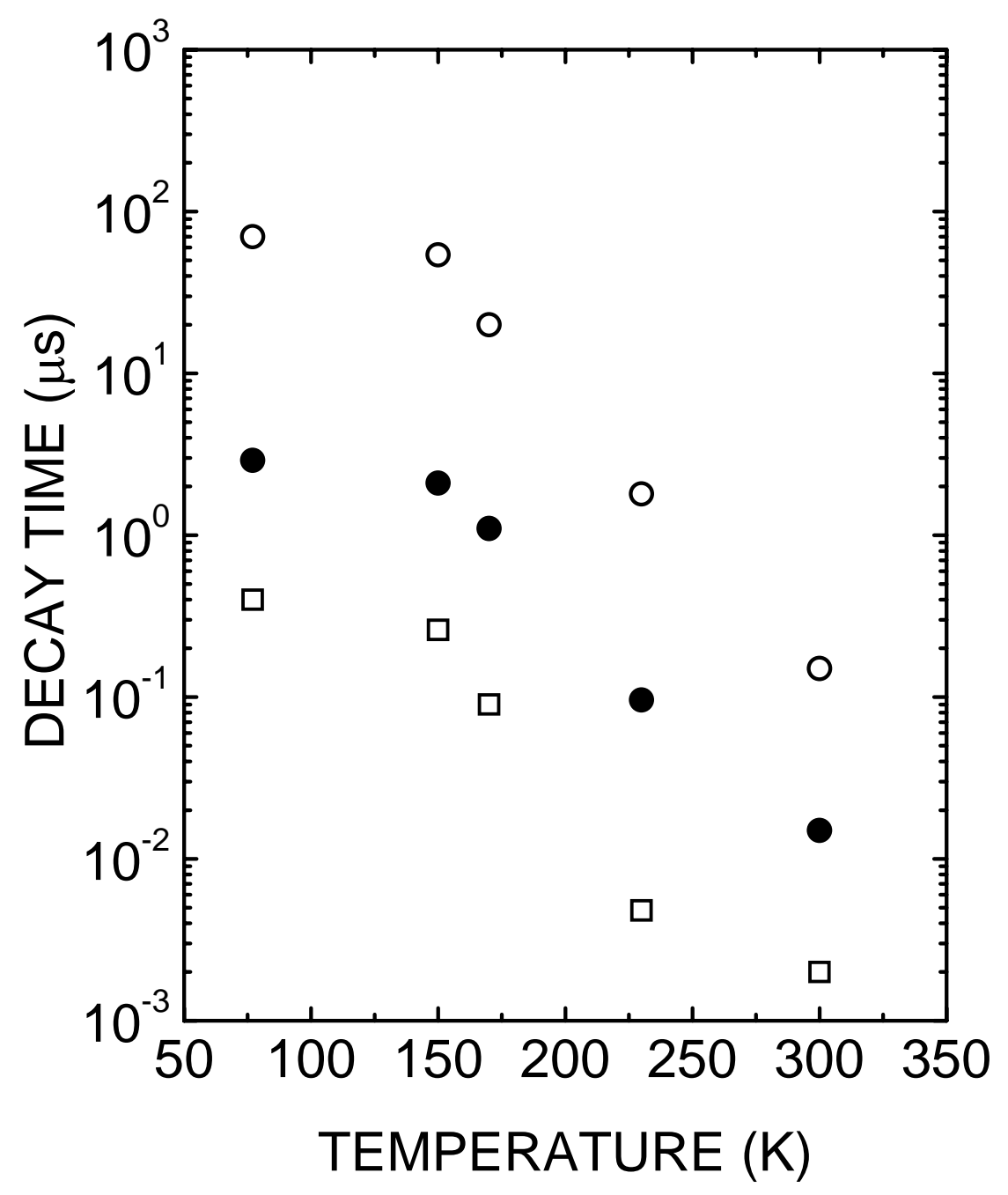

Fig. 3 


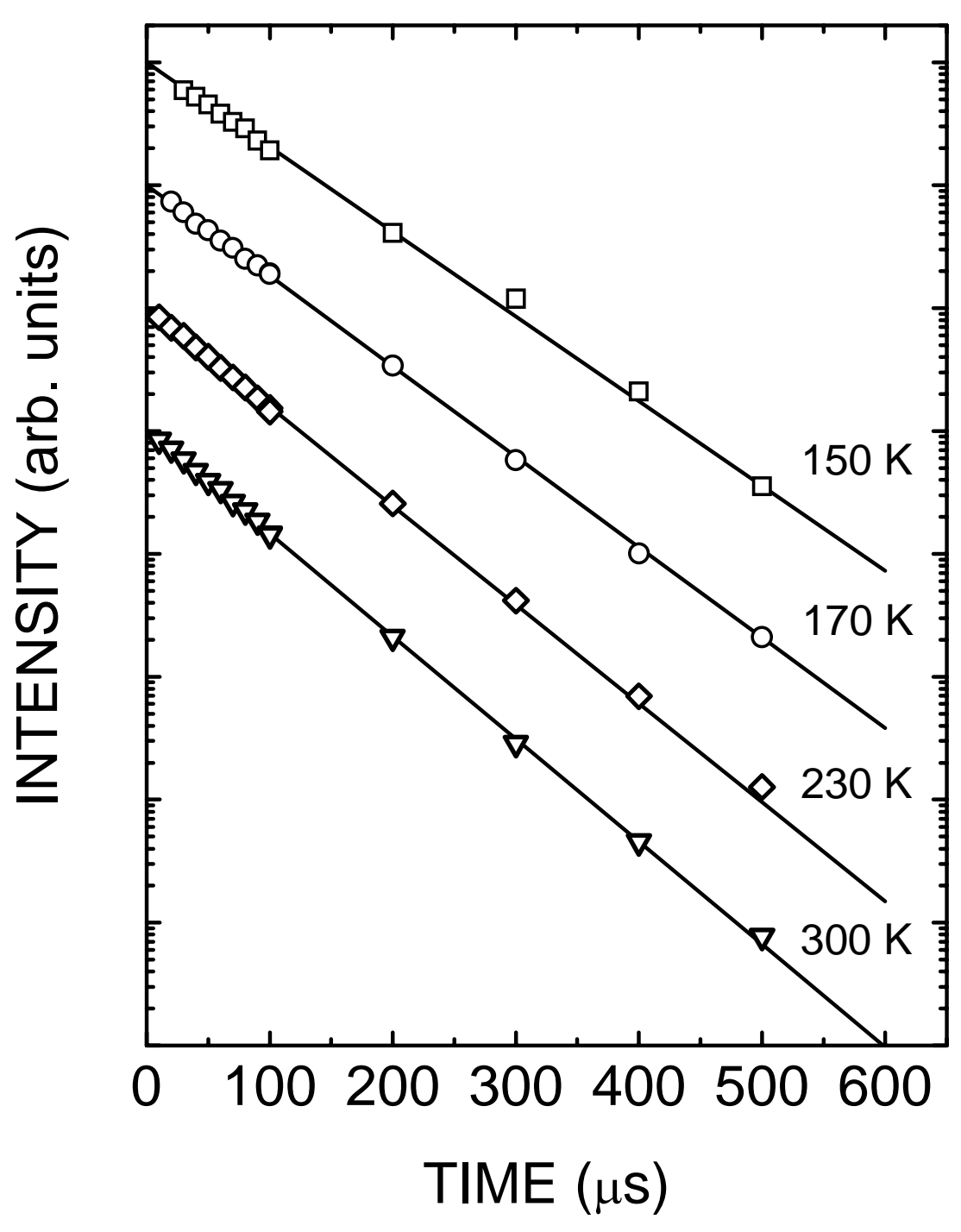

Fig. 4 


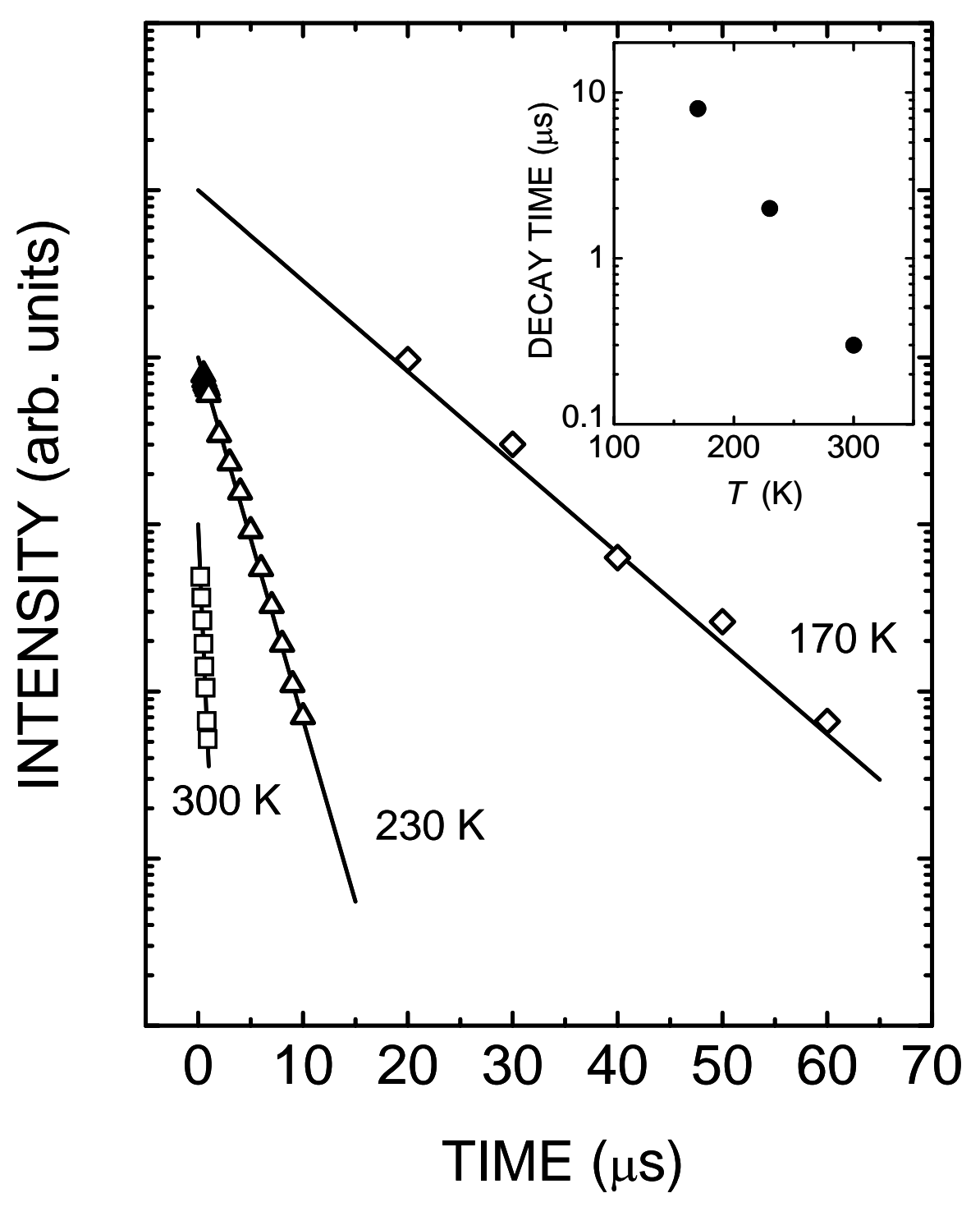

Fig. 5 


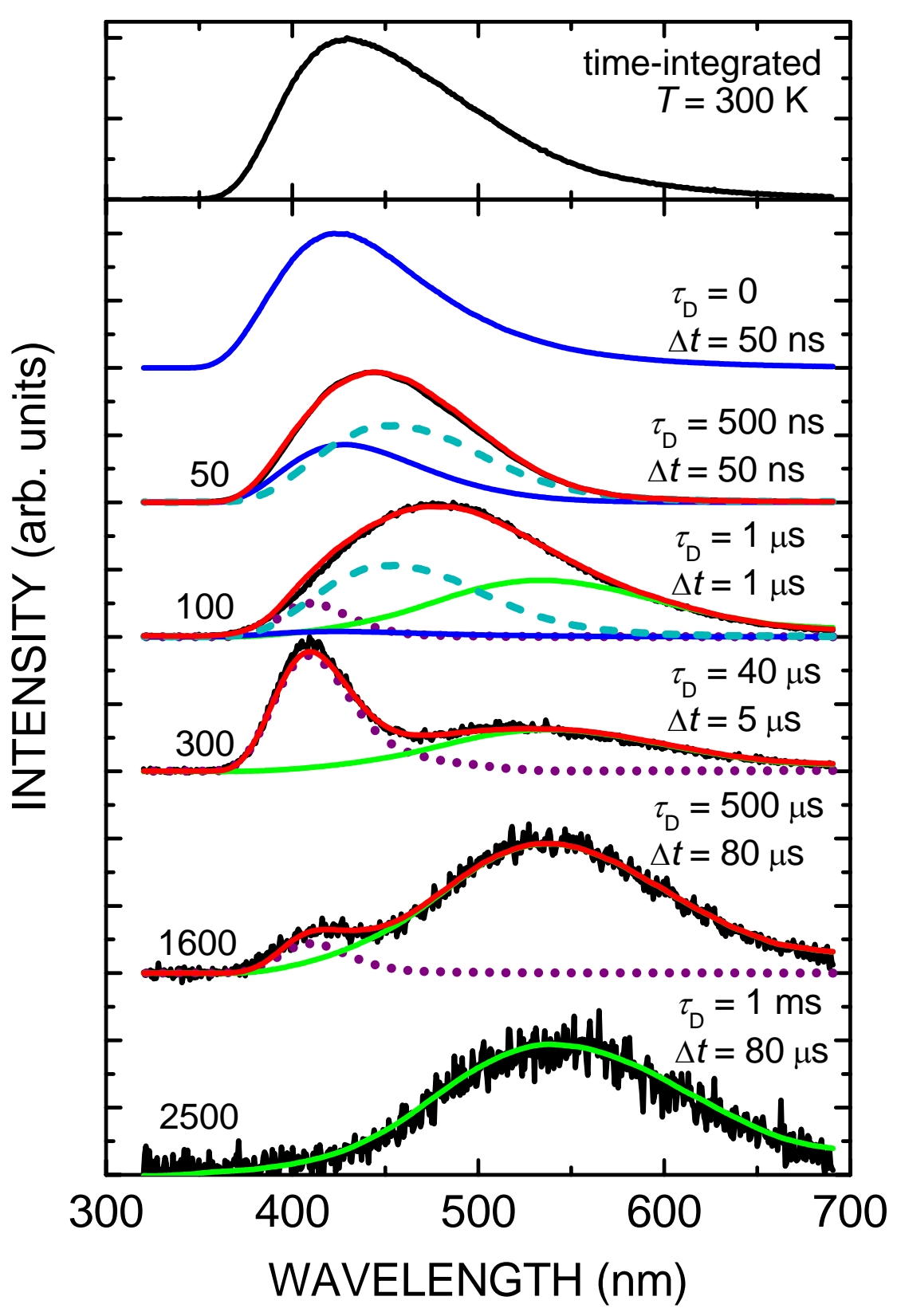

Fig. 6 
Published in: Phys. Rev. B. 73(23):235106 (8 J une 2006)

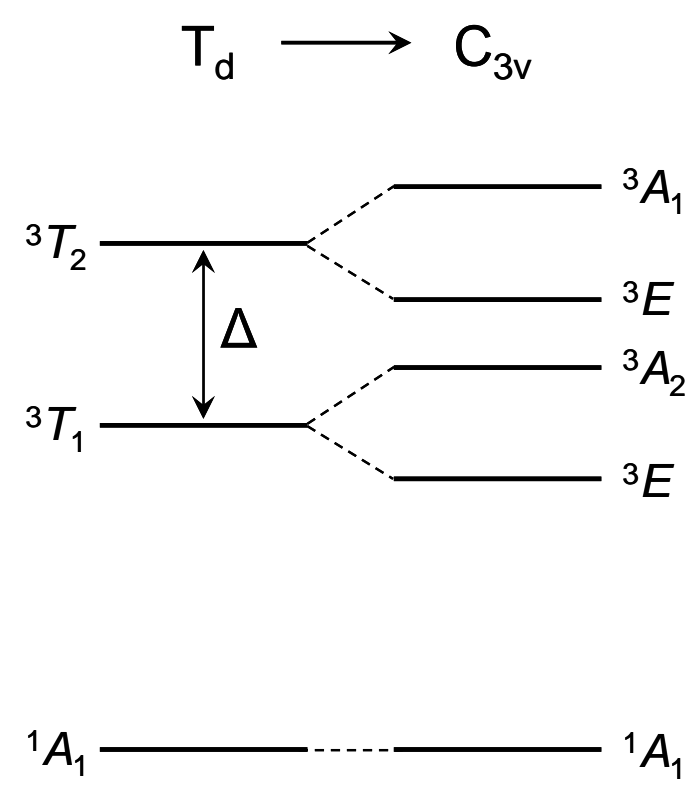

(a)
$\mathrm{T}_{\mathrm{d}} \longrightarrow \mathrm{C}_{3 \mathrm{v}}$

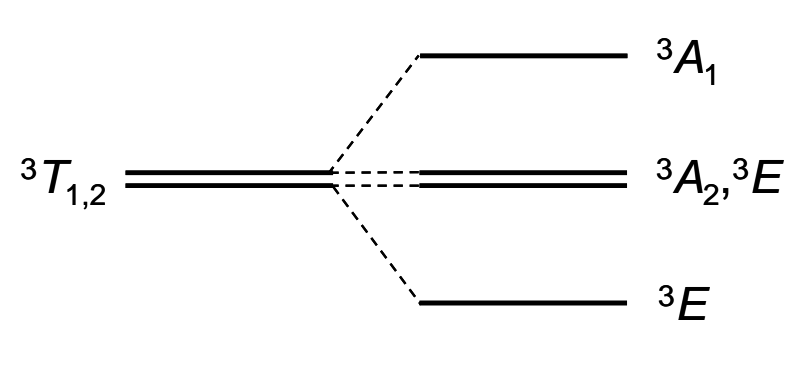

${ }^{1} A_{1}$

${ }^{1} A_{1}$

(b)

Fig. 7 


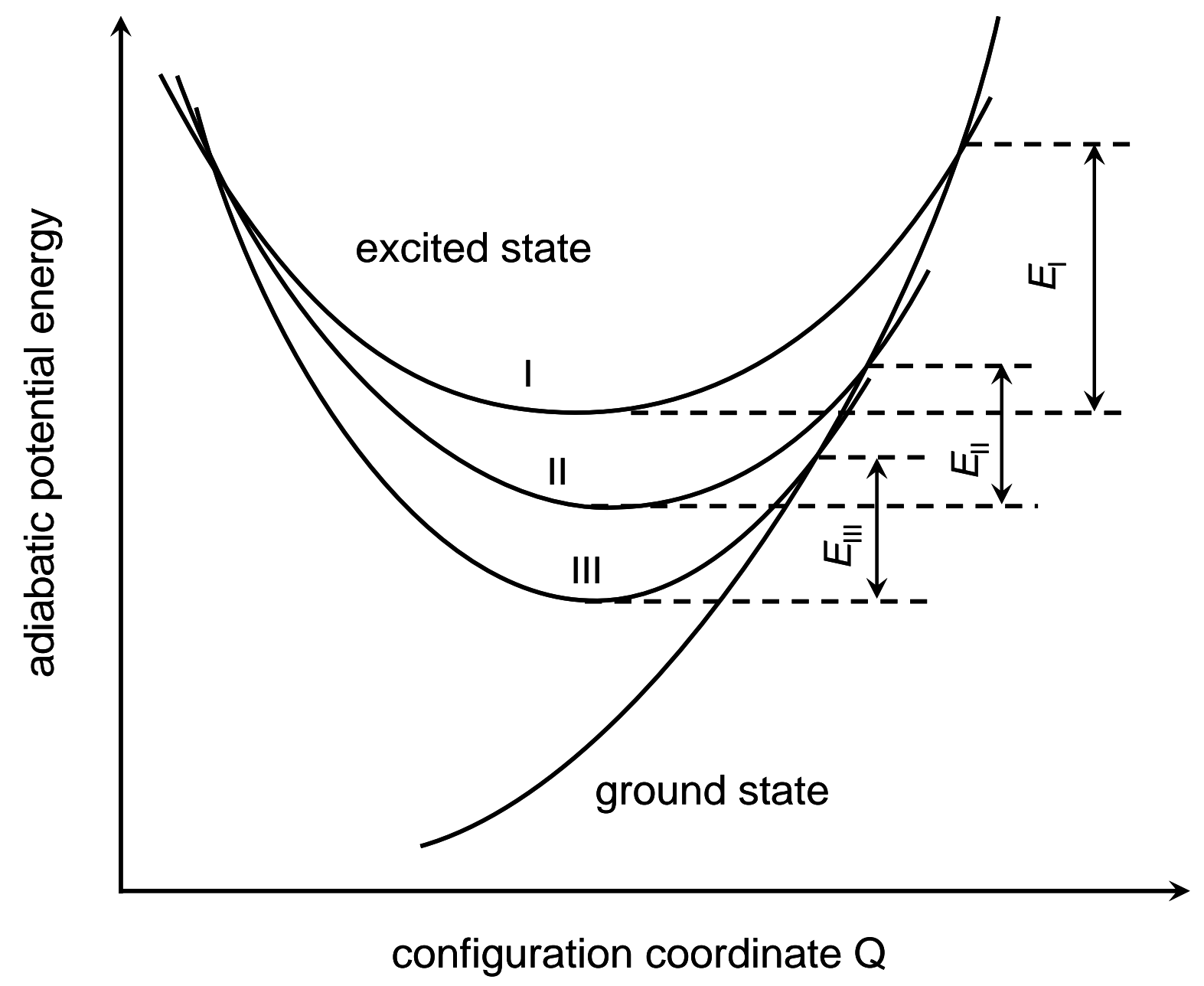

Fig. 8 